Proceeding Series of the Brazilian Society of Computational and Applied Mathematics

\title{
Técnicas de otimização combinatória aplicadas a um precondicionador paralelo baseado no algoritmo SPIKE
}

\author{
Brenno A. Lugon ${ }^{1}$, Leonardo M. Lima ${ }^{2}$, Marcelo T. P. Carrion ${ }^{1}$ \\ Lucia Catabriga $^{1}$, Maria Cristina Rangel ${ }^{1}$, Maria Claudia S. Boeres ${ }^{1}$ \\ Departamento de Informática, UFES, Vitória, ES
}

\begin{abstract}
Neste trabalho, utilizamos o algoritmo paralelo híbrido SPIKE como um precondicionador para um método iterativo não-estacionário combinando as arquiteturas de memória distribuída e compartilhada MPI/OpenMP. A fim de obter um bom precondicionador resolvemos um conjunto de problemas combinatórios como reordenamento, particionamento, matching e o problema quadrático da mochila. Apresentamos os resultados avaliando o speedup e escalabilidade em sistemas resultantes de formulações de elementos finitos.
\end{abstract}

Keywords. SPIKE, algoritmo paralelo híbrido, problemas combinatórios

\section{Introdução}

Com o crescimento na área de programação paralela e evolução das arquiteturas paralelas, uma tendência é o uso de uma abordagem híbrida MPI/OpenMP. Neste paradigma de programação, multicomputadores exploram o paralelismo usando memória distribuída enquanto multiprocessadores garantem as vantagens do uso de memória compartilhada. A Figura 1 exibe o esquema arquitetural dessa abordagem híbrida: diferentes ranks (Memória + CPU com 4 cores) se comunicam usando MPI via rede, enquanto as threads - ou cores - em cada rank se comunicam usando OpenMP.
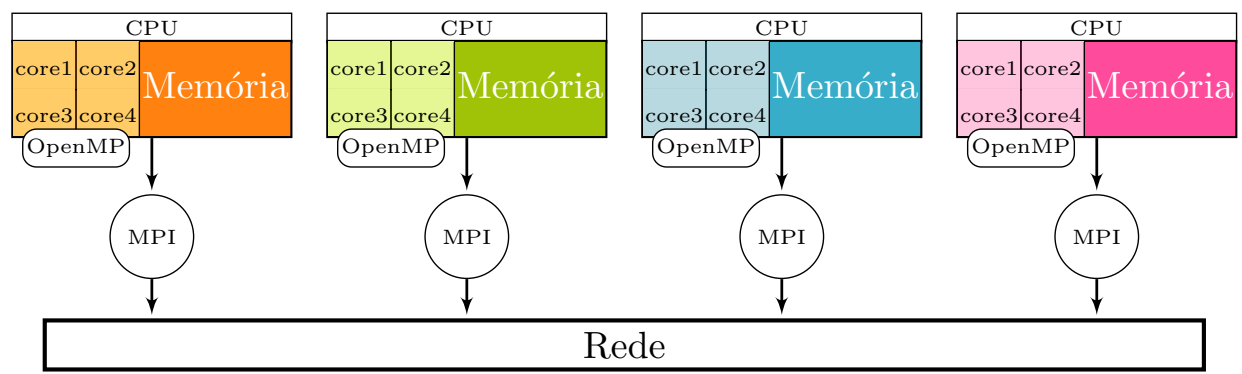

Figura 1: Abordagem híbrida MPI e OpenMP

\footnotetext{
${ }^{1}$ \{blugon,mcarrion,luciac,crangel,boeres\}@inf.ufes.br

${ }^{2}$ lmuniz@ifes.edu.br
} 
Para a resolução de sistemas lineares de grande porte, [10] sugere a utilização de métodos iterativos baseados em projeções de subespaços de Krylov por suas boas propriedades numéricas e computacionais. Além disso, o uso de precondicionadores acelera a convergência e consequentemente melhora a eficiência desses métodos na obtenção da solução. Em aplicações paralelas, o algoritmo híbrido SPIKE [8,9], criado inicialmente para resolver um sistema linear, pode também ser usado como precondicionador para o método iterativo. Ele é dito um método híbrido por ser capaz de tirar vantagem da robustez dos métodos diretos e do baixo custo computacional dos métodos iterativos.

Este artigo, cujo objetivo é a implementação de um método GMRES híbrido precondicionado com SPIKE considerando técnicas de otimização, está organizado da seguinte forma: na seção 2, explicamos o funcionamento do algoritmo SPIKE e as adaptações - e estratégias combinatórias — necessárias para usá-lo como precondicionador; a seção 3 apresenta os resultados obtidos de testes computacionais, com conclusões e trabalhos futuros sendo abordados na seção 4; por fim, seguem as seções de agradecimentos e referências bibliográficas.

\section{O algoritmo SPIKE}

O algoritmo paralelo híbrido SPIKE tem como objetivo encontrar a solução de um sistema linear da forma $A x=f$ usando computação paralela. Considere $A=\left\{a_{i j}\right\}_{n \times n}$ como sendo uma matriz esparsa com estrutura de banda, i.e., com os elementos nãonulos dispostos próximos à diagonal principal. Considere também $A$ como sendo diagonal dominante, ou seja, $\sum_{i \neq j}\left|a_{i j}\right| \leq\left|a_{i i}\right|, \forall i$. O algoritmo SPIKE se baseia na decomposição $A=D \times S$, onde $D$ é uma matriz bloco diagonal e $S=D^{-1} A$ é chamada de matriz de spikes. Portanto, resolver o sistema $A x=f$ recai em resolver os sistemas $D g=f$ e $S x=g$. A Figura 2 mostra como é feita essa decomposição para $p=3$ processadores.

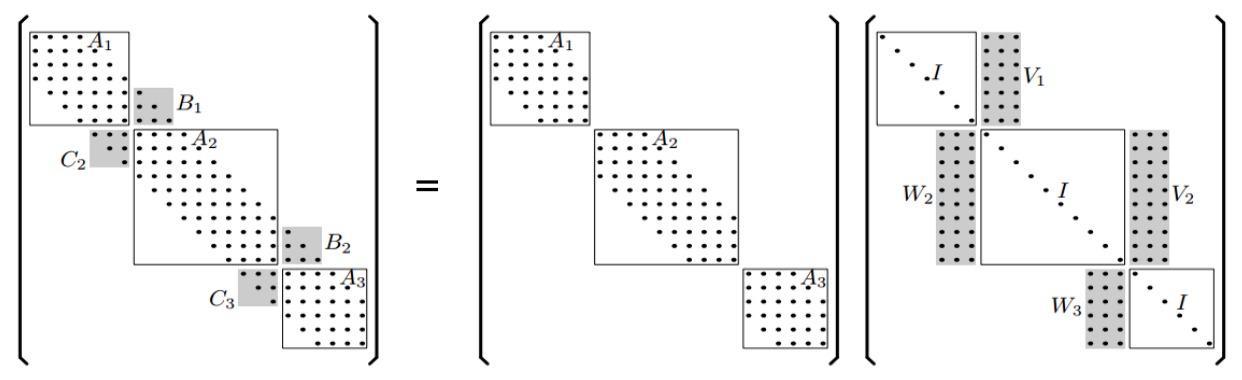

Figura 2: Decomposição $A=D \times S$

As matrizes $\mathbf{B}_{i}$ e $\mathbf{C}_{i+1}, i=1, \ldots, p-1$, são chamadas de blocos de acoplamento e possuem dimensão $\mathbf{k} \times \mathbf{k}$, onde $\mathbf{k}$ é a largura de banda de $A$. As matrizes $\mathbf{V}_{i}$ e $\mathbf{W}_{i+1}$, $i=1, \ldots, p-1$, são chamadas de spikes e formam a matriz de spikes $S$. De forma geral, as etapas do algoritmo SPIKE são:

1. Particionar o sistema original em vários ranks (ou processadores).

2. Aplicar a fatoração $\mathbf{L} \mathbf{U}$ em cada bloco diagonal $A_{i}$. 
3. Construir a matriz de spikes $S$ resolvendo:

$$
\begin{aligned}
\mathbf{L}_{1} \mathbf{U}_{1}\left[\mathbf{V}_{1}, \mathbf{g}_{1}\right] & =\left[\left(\begin{array}{c}
0 \\
\mathbf{B}_{1}
\end{array}\right), \mathbf{f}_{1}\right] \\
\mathbf{L}_{i} \mathbf{U}_{i}\left[\mathbf{W}_{i}, \mathbf{V}_{i}, \mathbf{g}_{i}\right] & =\left[\left(\begin{array}{c}
\mathbf{C}_{i} \\
0
\end{array}\right),\left(\begin{array}{c}
0 \\
\mathbf{B}_{i}
\end{array}\right), \mathbf{f}_{i}\right] \\
\mathbf{L}_{p} \mathbf{U}_{p}\left[\mathbf{W}_{p}, \mathbf{g}_{p}\right] & =\left[\left(\begin{array}{c}
\mathbf{C}_{p} \\
0
\end{array}\right), \mathbf{f}_{p}\right],
\end{aligned}
$$

onde $1<i<p$ sendo $p$ o número de processadores.

4. Montar e resolver o sistema reduzido $S z=g$ :

$$
\left[\begin{array}{cccc}
I & \mathbf{V}_{1}^{(b)} & & \\
\mathbf{W}_{i}^{(t)} & I & & \mathbf{V}_{i}^{(t)} \\
\mathbf{W}_{i}^{(b)} & & I & \mathbf{V}_{i}^{(b)} \\
& & \mathbf{W}_{p}^{(t)} & I
\end{array}\right]\left[\begin{array}{c}
z_{1}^{(b)} \\
z_{i}^{(t)} \\
z_{i}^{(b)} \\
z_{p}^{(t)}
\end{array}\right]=\left[\begin{array}{c}
g_{1}^{(b)} \\
g_{i}^{(t)} \\
g_{i}^{(b)} \\
g_{p}^{(t)}
\end{array}\right],
$$

onde $\mathbf{V}_{i}^{(b)}, \mathbf{W}_{i}^{(b)}$ e $\mathbf{V}_{i}^{(t)}, \mathbf{W}_{i}^{(t)}$ são a parte inferior e superior de tamanho $\mathbf{k} \times \mathbf{k}$ das matrizes $\mathbf{V}_{i}$ e $\mathbf{W}_{i}$, respectivamente. Da mesma forma, $z_{i}^{(b)}, g_{i}^{(b)}$ e $z_{i}^{(t)}, g_{i}^{(t)}$ são a parte inferior e superior de tamanho $\mathbf{k}$ dos vetores $z_{i}$ e $g_{i}$, respectivamente.

5. Uma vez que o sistema $S$ foi resolvido, a solução final pode ser obtida efetuando:

$$
\begin{aligned}
& x_{1}=g_{1}-V_{1} z_{2} \\
& x_{i}=g_{i}-W_{i} z_{i-1}-V_{i} z_{i+1} \quad \text { para } i=2, \ldots, p-1 \\
& x_{p}=g_{p}-W_{p} z_{p-1} .
\end{aligned}
$$

Com o objetivo de melhorar a eficiência - reduzindo os cálculos computacionais envolvidos na decomposição $\mathbf{L} \mathbf{U}$ - , podemos calcular apenas as partes superiores e inferiores de tamanho $\mathbf{k} \times \mathbf{k}$ das matrizes $\mathbf{W}_{i}, \mathbf{V}_{i}$ e do vetor $g_{i}$. Assim, a solução final é calculada por:

$$
\begin{array}{rlr}
\mathbf{L}_{1} \mathbf{U}_{1} x_{1} & =f_{1}-B_{1} z_{2} & \\
\mathbf{L}_{i} \mathbf{U}_{i} x_{i} & =f_{i}-C_{i} z_{i-1}-B_{i} z_{i+1} & \text { para } i=2, \ldots, p-1 \\
\mathbf{L}_{p} \mathbf{U}_{p} x_{p} & =f_{p}-C_{p} z_{p-1} . &
\end{array}
$$

\subsection{O algoritmo SPIKE como precondicionador}

O algoritmo SPIKE pode ser facilmente usado como um precondicionador dentro de um método iterativo não-estacionário como o GMRES ou BiCGStab [10]. Nesse caso, a matriz precondicionadora é dada por $M=\tilde{D} \tilde{S}$ da decomposição do SPIKE. A mudança com relação ao algoritmo original se dá na montagem do sistema reduzido $S z=g$ na etapa 4, como mostra a equação (5). Agora, podemos desconsiderar as matrizes $\mathbf{V}_{i}^{(t)}$ e $\mathbf{W}_{i}^{(b)}$ e também $\mathbf{k}$ pode ser menor que a largura de banda de $A$ - já que o precondicionador representa apenas uma aproximação $\bar{x}$ da solução final. O Algoritmo 1 mostra o laço principal do método iterativo com precondicionador SPIKE.

$$
\left[\begin{array}{cc}
I & \mathbf{V}_{i}^{(b)} \\
\mathbf{W}_{i+1}^{(t)} & I
\end{array}\right]\left[\begin{array}{c}
z_{i}^{(b)} \\
z_{i+1}^{(t)}
\end{array}\right]=\left[\begin{array}{c}
g_{i}^{(b)} \\
g_{i+1}^{(t)}
\end{array}\right]
$$




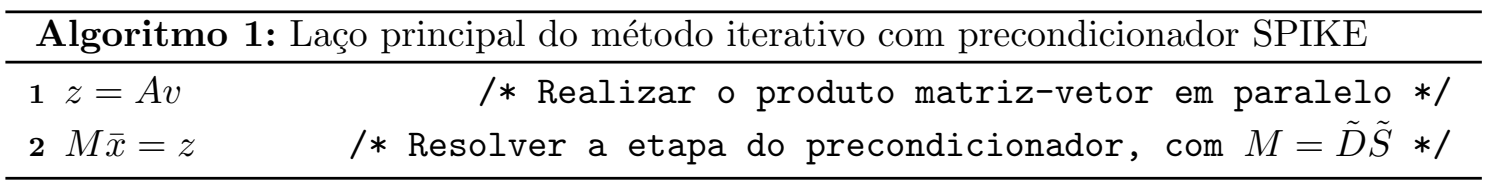

Neste trabalho, as etapas de cálculo dos fatores LU foram feitas usando o software PARDISO $[2,12,13]$ de resolução de sistemas lineares via métodos diretos usando memória compartilhada. De maneira eficiente, o PARDISO é capaz de resolver sistemas com múltiplos vetores de termos independentes, além de calcular somente as partes inferiores e superiores da solução de um sistema. Chamada de PSPIKE [3], a junção PARDISO + SPIKE é bastante eficiente na exploração do paralelismo MPI/OpenMP.

O uso do PARDISO não é a única maneira de se obter somente as partes inferiores e superiores das matrizes $\mathbf{W}_{i}, \mathbf{V}_{i}$ e do vetor $g_{i}$. Uma outra abordagem é o uso da estratégia $\mathbf{L U} / \mathbf{U L}$, onde se obtém $\mathbf{V}_{i}^{b}$ usando a fatoração $\mathbf{L U}$ e $\mathbf{W}_{i}^{t}$ através da fatoração $\mathbf{U L}$. Dessa forma, reduzem-se os custos computacionais pois não é necessário calcular todos os coeficientes de cada fatoração [5].

\section{$2.2 \quad$ Estratégias combinatórias}

Para uma matriz A qualquer ser resolvida pelo SPIKE, é necessário transformá-la através de permutações de linhas e colunas - em uma matriz com estrutura de banda, tal que suas entradas sejam cobertas pelas blocos diagonais $A_{i}$ e pelos blocos de acoplamento $B_{i}$ e $C_{i+1}$. Um bom precondicionador $M$ deve contemplar nesses blocos os elementos não-nulos de maior valor absoluto. De forma geral, quatro estratégias de otimização combinatória são aplicadas na matriz, visando satisfazer quatro objetivos principais:

(i) Tornar a matriz original em uma matriz com estrutura de banda;

(ii) Obter os blocos diagonais;

(iii) Garantir a não-singularidade de cada bloco diagonal;

(iv) Mover elementos mais significativos para os blocos de acoplamento.

Cada objetivo é modelado como um problema combinatório, como mostrado a seguir.

\subsubsection{Reordenamento}

O reordenamento de matrizes esparsas é um problema bastante estudado e, no contexto deste trabalho, seu objetivo é transformar uma matriz geral em uma matriz com estrutura de banda, aproximando os elementos não-nulos da diagonal principal. Ele pode ser visto como um problema de rerrotulação de grafos, para o qual adotamos o algoritmo Reverse Cuthill-McKee (RCM) [1] pelos bons resultados presentes na literatura.

\subsubsection{Particionamento}

Nesse problema, desejamos atribuir linhas/colunas consecutivas da matriz a diferentes processadores, i.e., determinar as matrizes $A_{i}$, equilibrando o número de elementos nãonulos em cada um deles. Esse particionamento é modelado como um problema específico, chamado de Chains-on-chains partitioning (CCP) [7]. O CCP pode ser resolvido em tempo polinomial, e o algoritmo MinMax [4] é empregado para obter a solução exata. 


\subsubsection{Matching}

O problema de estabelecer que os blocos diagonais $A_{i}$ sejam não-singulares e, portanto, possam ser resolvidos utilizando a fatoração $\mathbf{L} \mathbf{U}$ em cada processador, pode ser interpretado como um problema de matching valorado em grafos. O objetivo é permutar linhas no bloco de modo que elementos de grande valor absoluto fiquem na diagonal. Isso equivale a obter, no grafo bipartido que representa $A_{i}$, um matching perfeito de valor máximo, com o Successive Shortest Path algorithm [6] sendo usado para essa tarefa.

\subsubsection{Problema quadrático da mochila}

Consiste em obter os blocos de acoplamento $B_{i}$ e $C_{i+1}$ de tamanho $\mathbf{k} \times \mathbf{k}$, através de uma permutação simétrica (para não alterar os efeitos da etapa anterior) da matriz. De modo geral, permutam-se linhas e colunas de forma a concentrar elementos mais significativos em módulo - localizados nas submatrizes definidas entre os blocos diagonais $A_{i}$ - dentro dos blocos de acoplamento. A heurística DeMin proposta por [11] é aplicada para realizar tal reordenamento.

\section{Testes computacionais}

Foi realizado um conjunto de testes com matrizes derivadas de problemas de elementos finitos, como mostra a Tabela 1. Os testes foram executados no cluster Enterprise 3 do laboratório LCAD na Ufes. Ele possui 28 máquinas quad-core Intel 2 Q6600 (116 cores) com $2.4 \mathrm{GHz}$ de frequência de clock, $4 \mathrm{MB}$ L2 e $4 \mathrm{~GB}$ of DRAM, interconectadas por um switch 48-Port 4200G 3COM Gigabit Ethernet. Os códigos em C foram compilados com gcc 4.4.7, usando Open MPI 1.6.2, no sistema operacional CentOS 6.5. A versão da biblioteca PARDISO utilizada foi pardiso500-MPI-GNU450-X86-64.

\begin{tabular}{lrrr}
\hline matriz & dimensão $(\mathrm{n})$ & não-nulos $(\mathrm{nnz})$ & área de aplicação \\
\hline mesh_1 & 1.043 .474 & 7.294 .192 & Elementos Finitos 2D \\
cube_1 & 83.987 & 1.277 .909 & Elementos Finitos 3D \\
\hline
\end{tabular}

Tabela 1: Características das matrizes-teste

\begin{tabular}{|c|c|c|c|c|c|c|c|c|}
\hline & \multicolumn{2}{|r|}{ GMRES } & \multicolumn{3}{|c|}{$\mathrm{P}_{\mathrm{S}}$ GMRES, $\mathbf{k}=50$} & \multicolumn{3}{|c|}{$\mathrm{P}_{\mathrm{S}}$ GMRES, $\mathbf{k}=100$} \\
\hline & \multirow[b]{2}{*}{ MPI } & & \multicolumn{3}{|c|}{ OpenMP } & \multicolumn{3}{|c|}{ OpenMP } \\
\hline & & & 1 & 2 & 4 & 1 & 2 & 4 \\
\hline \multirow{4}{*}{ 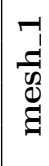 } & 2 & 2503.06 & 123.68 & 94.91 & 89.05 & 132.53 & 103.54 & 95.65 \\
\hline & 4 & 1427.71 & 89.74 & 70.60 & 65.86 & 101.44 & 80.24 & 75.55 \\
\hline & 8 & 865.57 & 50.05 & 39.65 & 37.18 & 58.21 & 47.31 & 44.27 \\
\hline & 16 & 541.26 & 27.49 & 25.86 & 20.65 & 38.22 & 35.47 & 30.40 \\
\hline \multirow{4}{*}{$\begin{array}{l}7 \\
0 \\
0 \\
0 \\
0\end{array}$} & 2 & 2714.71 & 38.53 & 24.07 & 19.50 & 47.74 & 29.20 & 22.88 \\
\hline & 4 & 1945.78 & 13.14 & 8.92 & 7.79 & 18.15 & 12.11 & 10.22 \\
\hline & 8 & 1832.29 & 5.79 & 4.38 & 4.23 & 8.47 & 6.58 & 5.91 \\
\hline & 16 & 1538.70 & 3.64 & 3.01 & 2.85 & 5.46 & 4.93 & 4.42 \\
\hline
\end{tabular}

Tabela 2: Diferentes tempos (em segundos) para resolver cada matriz-teste 
Os tempos computacionais, em segundos, de testes preliminares para resolução dos sistemas oriundos das matrizes-teste são exibidos na Tabela 2. A coluna GMRES mostra o caso não-precondicionado, enquanto as colunas rotuladas com $P_{S}$ GMRES, $\mathbf{k}=50 \mathrm{e}$ $\mathrm{P}_{\mathrm{S}}$ GMRES, $\mathbf{k}=100$ se referem aos testes precondicionados com SPIKE, sendo $\mathbf{k}$ o tamanho dos blocos de acoplamento arbitrado em cada caso. Para cada matriz, as diferentes linhas indicam quantidades distintas de ranks MPI (descritas na coluna MPI) utilizados na parte de computação distribuída, enquanto as colunas identificadas com 1, 2 e 4 consideram o correspondente número de threads OpenMP usadas pelo PARDISO. Em ambas as matrizes, provou-se ser mais vantajoso combinar o maior número de ranks ao maior número de threads, ou seja, 16 ranks MPI e 4 threads OpenMP.

A Figura 3 apresenta os gráficos dos speedups referentes aos testes realizados com as matrizes-teste somente considerando $\mathbf{k}=50$ (que obteve melhores resultados). O speedup alcançado por mesh_1 é bem parecido ao serem consideradas 1, 2 ou 4 threads OpenMP, o que sugere uma certa escalabilidade. Já o gráfico da matriz cube_1 apresenta alguns pontos de speedup superlinear, que possivelmente não seria sustentado ao se empregar mais ranks MPI como a própria curva sugere. O tamanho de cube_1 e sua forma, ou seja, menor e mais densa, podem haver influenciado a hierarquia da memória cache.
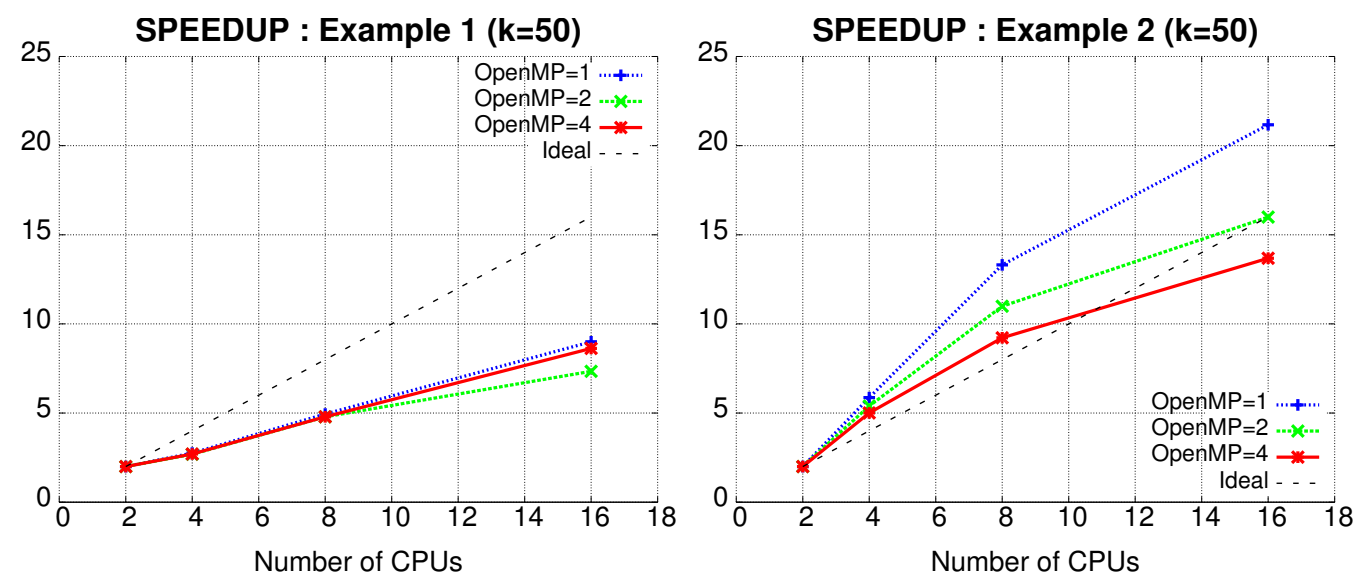

Figura 3: Speedup das matrizes mesh_1 (à esquerda) e cube_1 (à direita)

\section{Conclusões e trabalhos futuros}

O desempenho do precondicionador SPIKE depende fortemente da qualidade de transformação da matriz original $A$ através das operações combinatórias apresentadas, e especialmente da escolha do tamanho k. Quando $\mathbf{k}$ é maior, o número de iterações do método precondicionado tende a diminuir, uma vez que mais elementos da matriz transformada estão sendo considerados. Entretanto, essa diminuição raramente conduz a uma redução do tempo computacional total, pois as operações de decomposição LU e comunicações MPI relacionadas aos blocos $\mathbf{V}_{i}$ e $\mathbf{W}_{i}$ ficam mais custosas.

Como trabalhos futuros, propõe-se uma implementação própria das funções da biblioteca PARDISO inseridas no contexto do algoritmo SPIKE. Além disso, pretende-se utilizar esse precondicionador em plataformas de computação de larga escala e aplicar uma abordagem paralela às técnicas combinatórias aqui empregadas na forma sequencial. 


\section{$5 \quad$ Agradecimentos}

Os autores agradecem às agências de fomento FAPES, CAPES e CNPq o apoio recebido através de bolsas de estudo e suporte financeiro na realização de projetos de pesquisa.

\section{Referências}

[1] E. Cuthill and J. McKee, Reducing the bandwidth of sparse symmetric matrices, Proceedings of the 1969 24th National Conference, 157-172, (1969).

[2] A. Kuzmin, M. Luisier and O. Schenk, Fast methods for computing selected elements of the greens function in massively parallel nanoelectronic device simulations, Lecture Notes in Computer Science, vol. 8097, 533-544, (2013).

[3] M. Manguoglu, A. Sameh and O. Schenk, Pspike: A parallel hybrid sparse linear system solver, Lecture Notes in Computer Science, vol. 5704, 797-808, (2009).

[4] F. Manne and T. Sørevik, Optimal partitioning of sequences, Journal of Algorithms, vol. 19, 235-249, (1995).

[5] K. Mendiratta, A banded spike algorithm and solver for shared memory architectures, Master's thesis, (2011).

[6] J. B. Orlin and Y. Lee, Quickmatch-a very fast algorithm for the assignment problem, Working papers 3547-93., MIT, Sloan School of Management, (1993).

[7] A. Pinar and C. Aykanat, Fast optimal load balancing algorithms for 1d partitioning, Journal of Parallel and Distributed Computing, vol. 64, 974-996, (2004).

[8] E. Polizzi and A. Sameh, A parallel hybrid banded system solver: The spike algorithm, Parallel Computing, vol. 32, 177-194, (2006).

[9] E. Polizzi and A. Sameh, SPIKE: A parallel environment for solving banded linear systems, Computers \& Fluids, vol. 36, 113-120, (2007).

[10] Y. Saad, Iterative Methods for Sparse Linear Systems, SIAM, Philadelphia, 2nd ed., (2003).

[11] M. Sathe, O. Schenk, B. Uçar and A. Sameh, A Scalable Hybrid Linear Solver Based on Combinatorial Algorithms, Combinatorial Scientific Computing, ChapmanHall/CRC Computational Science, 95-128, (2012).

[12] O. Schenk, M. Bollhöfer and R. A. Römer, On large-scale diagonalization techniques for the anderson model of localization, SIAM Review, vol. 50, 91-112, (2008).

[13] O. Schenk, A. Wächter and M. Hagemann, Matching-based preprocessing algorithms to the solution of saddle-point problems in large-scale nonconvex interior-point optimization, Computational Optimization and Applications, vol. 36, 321-341, (2007). 